\title{
Modeling of spatially correlated energetic disorder in organic semiconductors
}

\author{
Pascal Kordt* and Denis Andrienko* \\ Max Planck Institute for Polymer Research, Ackermannweg 10, 55128 Mainz, Germany \\ E-mail: kordt@mpip-mainz.mpg.de; denis.andrienko@mpip-mainz.mpg.de
}

\begin{abstract}
Mesoscale modeling of organic semiconductors relies on solving an appropriately parametrized master equation. Essential ingredients of the parametrization are site energies, or driving forces, which enter the charge transfer rate between pairs of neighboring molecules. Site energies are often Gaussian-distributed and are spatially correlated. Here we propose an algorithm which generates these energies with a given Gaussian distribution and spatial correlation function. The method is tested on an amorphous organic semiconductor, DPBIC, illustrating that the accurate description of correlations is essential for the quantitative modeling of charge transport in amorphous mesophases.
\end{abstract}

today

\section{Introduction}

Early models of charge transport in organic semiconductors, such as the Gaussian disorder model (GDM), employed simple lattices in order to describe material morphology, postulated a Gaussian

\footnotetext{
*To whom correspondence should be addressed
} 
distribution of uncorrelated site energies driving charge transfer reactions, and used the thermallyactivated tunneling (Miller-Abrahams) ${ }^{1}$ rate expression to compute charge transfer rates. ${ }^{2}$ Material properties were thus represented by a small number of essential parameters: the width of the density of states, the lattice spacing, and the hopping attempt frequency. In spite of its simplicity, the GDM could already explain the non-trivial dependencies of the charge carrier mobility on the external electric field, temperature, and the charge carrier density. ${ }^{2-5}$

The GDM has been gradually refined in order to improve the agreement with experiment. First, it has been shown that the long-range electrostatic interactions of a charge with molecular dipoles $^{6,7}$ as well as a local alignment of conjugated segments in polymeric systems ${ }^{8,9}$ can lead to spatial correlations of site energies. By accounting for these correlations, the (extended) correlated Gaussian disorder models (ECDM, CDM), ${ }^{3,6}$ could explain the experimentally observed Poole-Frenkel dependence, $\ln \mu \propto \sqrt{F}$, also for small external fields. ${ }^{6,10}$ Second, a more direct link to the underlying chemical composition of the material could be established by combining quantum-chemical and classical force-field-based methods, which have been used to predict material morphologies and evaluate charge transfer rates. ${ }^{11-14,14,15}$ The atomic-scale modeling also stimulated the development of off-lattice stochastic models. ${ }^{16,17}$ In these models, molecular positions and rates are generated according to the distribution functions evaluated in a small atomistic morphology. Stochastic models allow to simulate charge transport in micrometer-thick layers while at the same time retaining the link to the chemical composition.

Both stochastic and atomistic models have helped to pinpoint drawbacks of GDM and CDM. In particular, the $\mathrm{CDM}$ assumes a charge interacting with randomly oriented, constant magnitude dipoles of surrounding molecules, ${ }^{7,18}$ which leads to an energy correlation function of the form $c(d) \sim 1 / d$. The underlying assumption is, however, not well justified: Figure Figure 1 shows that the distribution of magnitudes of molecular dipole moments in an amorphous mesophase can be rather broad. A similar situation has been observed in other systems, e.g., typical dye molecules used as donors in organic solar cells. ${ }^{19}$ As a consequence, the spatial correlations of site energies can have decays of the correlation function different from $1 / d$ dependence. ${ }^{12,19}$ 
For accurate parametrizations of stochastic models it is therefore desirable to have an algorithm which can generate site energies with a predefined correlation function. The goal of this work is to devise such an algorithm and to test it on a typical amorphous semiconductor, DPBIC, the chemical structure of which is shown in the inset of Figure Figure 1.

\section{Atomistic simulations}

To evaluate the reference spatial correlation function, we first use atomistic molecular dynamics to generate an amorphous morphology of 4000 DPBIC molecules. We then employ quantumchemical calculations and polarizable force-fields to evaluate electronic couplings, site and reorganization energies. To validate the algorithm and to illustrate the vital role of correlations, we finally study charge dynamics by solving the master equation with the help of the kinetic Monte Carlo algorithm. Charge hopping rates entering the master equation are evaluated within the hightemperature limit of the non-adiabatic charge transfer theory.

For morphology simulations we use an OPLS-based ${ }^{22,23}$ force-field with missing bonded interactions parametrized using the potential energy surface scans obtained using the density functional theory (B3LYP functional and 6-311g(d,p) basis set), as described elsewhere. ${ }^{17}$ Partial charges are fitted using the Merz-Kollman scheme. ${ }^{24,25}$ To obtain the amorphous morphology, the box of 4000 molecules is first simulated at $700 \mathrm{~K}$, which is above the glass transition temperature, and then quenched to $300 \mathrm{~K}$. The size of the final box is about $16 \mathrm{~nm}$. All calculations are performed in the $N P T$ ensemble with the Berendsen barostat and thermostat. ${ }^{26}$

The hole site energies are evaluated using a perturbative approach, in which electrostatic and induction energies are added to the gas-phase ionization potential of a molecule $A$, i.e., $E^{A}=E_{\mathrm{int}}^{A}+$ $E_{\mathrm{el}}^{A}+E_{\mathrm{pol}}^{A}$, where $E_{\mathrm{int}}^{A}$ is the ionization potential in vacuum, $E_{\mathrm{el}}^{A}$ is the electrostatic interaction energy of partial charges, and $E_{\mathrm{pol}}^{A}$ is the polarization contribution. Since rates depend only on site energy differences, ${ }^{1,27,28}$ the ionization potential does not affect rates or the spatial correlation function of a mono-component system. The electrostatic contribution to the site energies, $E_{\mathrm{el}}^{A}$, is calculated 


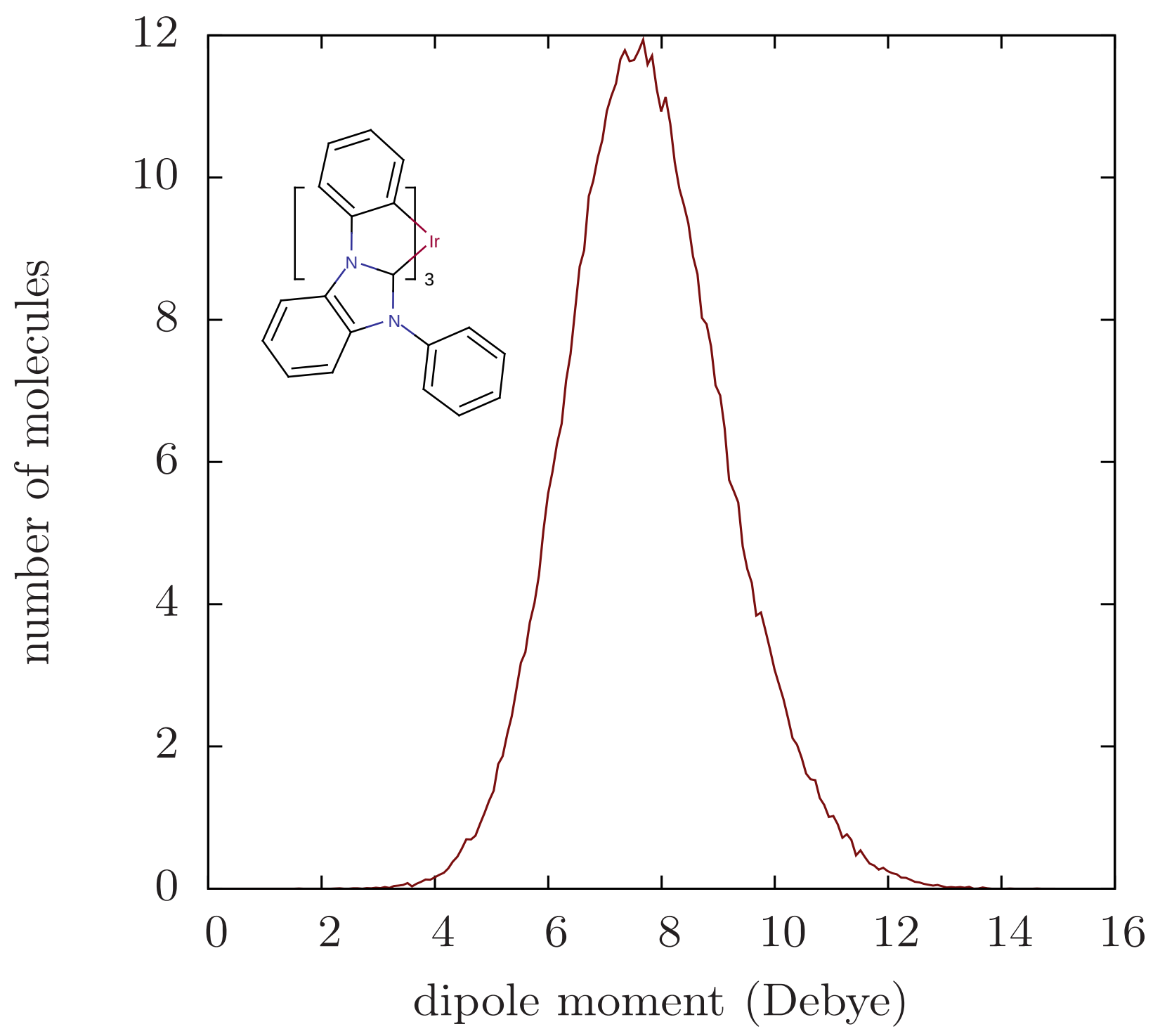

Figure 1: Distribution of molecular dipoles in an atomistic morphology of 4000 DPBIC molecules. The inset shows the chemical structure of DPBIC (Tris[(3-phenyl-1H-benzimidazol-1-yl-2(3H)ylidene)-1,2-phenylene]Ir), which is used as a hole-conducting and an electron-blocking layer in modern OLEDs. ${ }^{20,21}$ 


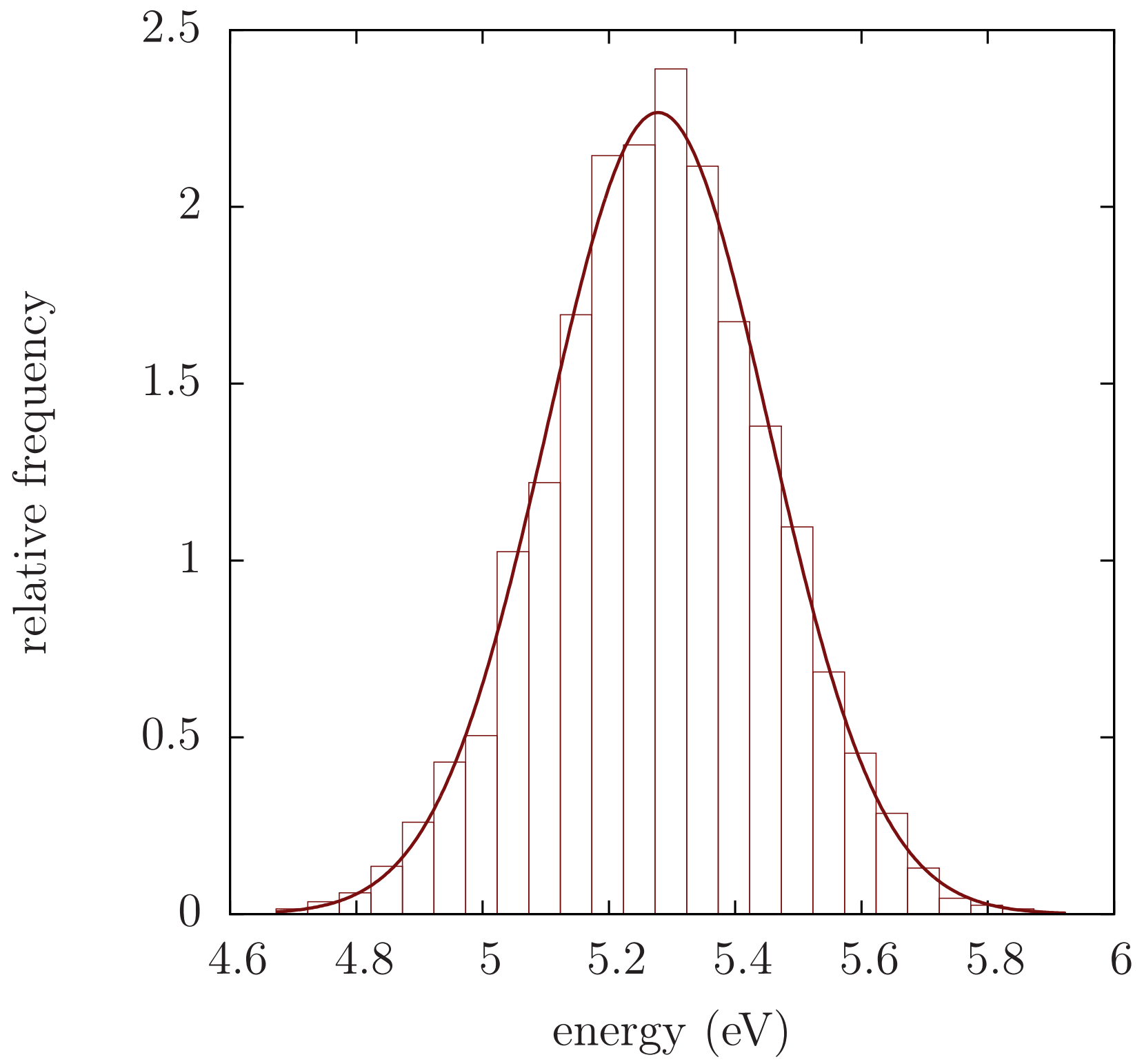

Figure 2: Site energy distribution evaluated in the atomistic morphology and the fit to a Gaussian distribution with $\sigma=0.176 \mathrm{eV}$. 


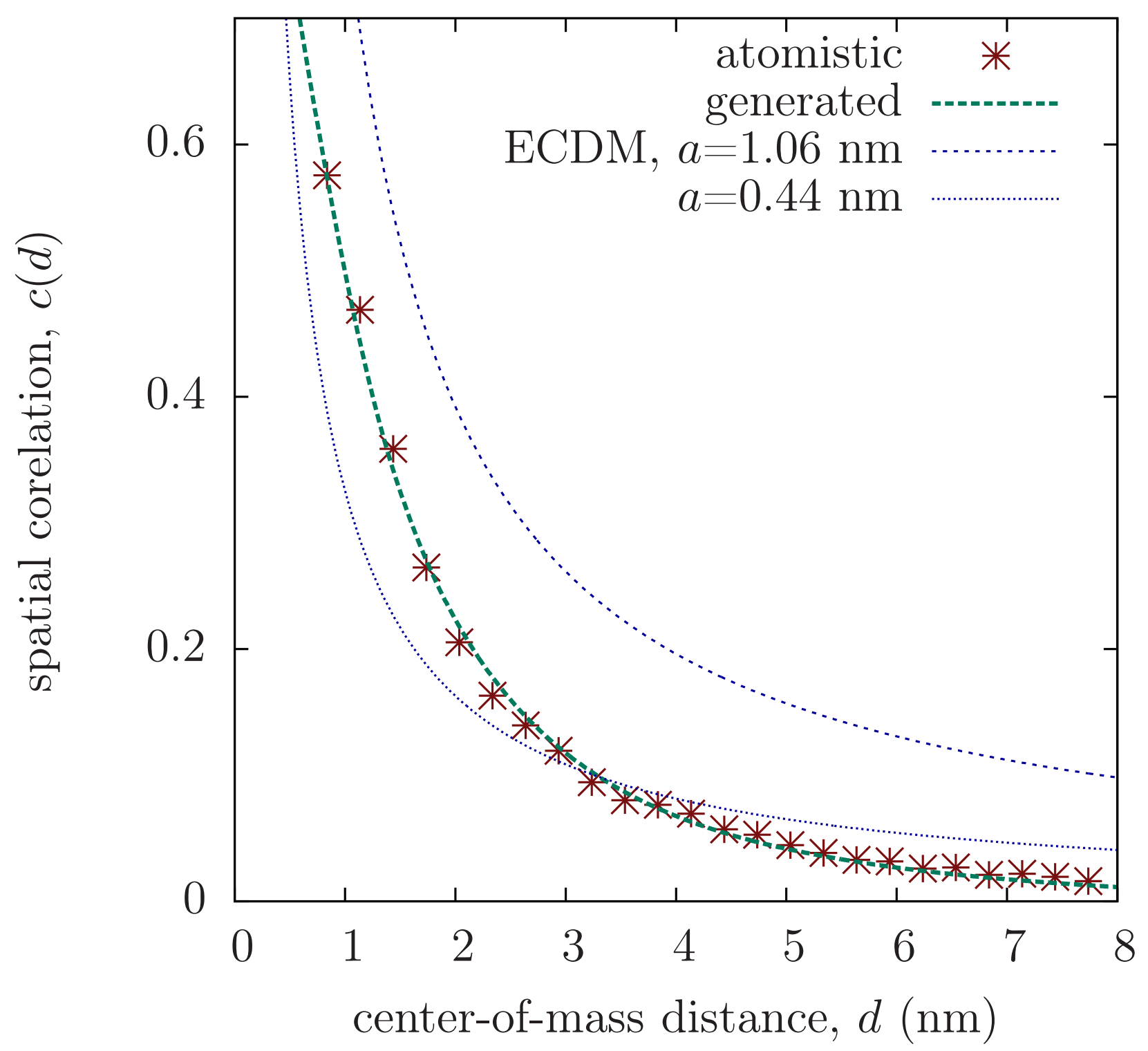

Figure 3: Spatial site energy correlation evaluated in an atomistic morphology (symbols) and its reconstruction using the numerical scheme described in the text (dashed line). Thin dotted lines show correlation functions of the ECDM model for two different lattice spacings. 
using the Ewald summation technique adapted for charged systems. ${ }^{29,30}$ All induction effects are incorporated in this scheme using the Thole model ${ }^{31,32}$ with a cutoff of $3 \mathrm{~nm}$. Details of this approach are described elsewhere. ${ }^{33}$ The distribution of site energies, shown in Figure Figure 2, is, to a good accuracy, Gaussian with a standard deviation of $\sigma=0.176 \mathrm{eV}$.

The spatial correlation function, $c(d)$, which describes the correlation of site energies of molecules at a (center-of-mass) separation $d$ is calculated as

$$
c(d)=\mathbb{E}\left[E^{A} E^{B}\right] / \sigma^{2},
$$

where $E^{A}$ and $E^{B}$ are energies of molecules $A$ and $B$ separated by distance $d$ and $\mathbb{E}[\cdot]$ is the expectation value. The spatial correlation $c^{\text {ref }}(d)$, evaluated in the atomistic system, is shown in Figure Figure 3 (symbols).

Since we are dealing with amorphous materials, we assume thermally-activated type of transport and use the semi-classical charge transfer rate expression ${ }^{27,28}$

$$
\omega_{A B}=\frac{2 \pi}{\hbar} \frac{J_{A B}^{2}}{\sqrt{4 \pi \lambda k_{\mathrm{B}} T}} \exp \left[-\frac{\left(\Delta E^{A B}-\lambda\right)^{2}}{4 \lambda k_{\mathrm{B}} T}\right]
$$

Here the reorganization energy, $\lambda$, reflects the effect of molecular rearrangement in response to the change of the charge state. $J_{A B}$ describes the strength of the electronic coupling between two localized (diabatic) states, and $\Delta E^{A B}=E^{A}-E^{B}$ is the aforementioned driving force. For DPBIC the calculated (B3LYP, 6-311g(d,p)) internal reorganization energy for hole transport is $\lambda=0.13 \mathrm{eV}$.

Electronic coupling elements, $J_{A B}$, between the initial and the final states of a molecular dimer are evaluated by approximating its diabatic sates with the highest occupied molecular orbitals of monomers (frozen core approximation) and using a projection method. ${ }^{34-36}$

To model charge dynamics, we solve the corresponding master equation using the kinetic 
Monte Carlo (KMC) algorithm. ${ }^{37,38}$ The mobility is calculated from the KMC trajectory as ${ }^{17}$

$$
\mu=\frac{1}{N} \sum_{i=1}^{N} \frac{\left\langle\vec{v}_{i} \cdot \vec{F}\right\rangle}{F^{2}}
$$

where $\vec{v}_{i}$ is the velocity of the $i$-th carrier, $\langle\cdots\rangle$ denotes the average over the simulation time, and the sum is performed over $N$ carriers.

\section{The algorithm}

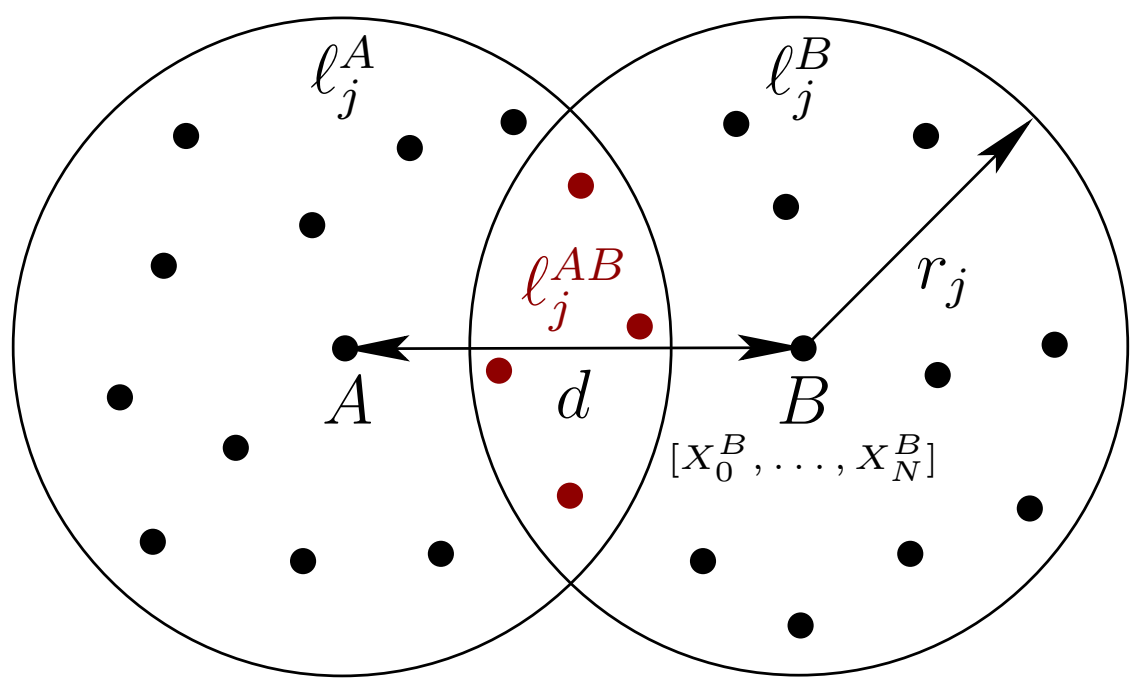

Figure 4: Mixing-in of site energies: the site energy correlation of two molecules $A$ and $B$ is determined by the (weighted) number of molecules, $\ell_{j}^{A B}$ (red), within the overlap of spheres of radii $r_{j}$ centered at $A$ and $B$.

Given the spatial correlation function, $c^{\mathrm{ref}}(d)$, and the variance, $\sigma^{2}$, of the Gaussian site energy distribution in the atomistic system, our goal is to generate site energies for a large stochastic morphology, with the same distribution and spatial correlation. The idea is to add to a site energy of a molecule $A$ appropriately weighted energies of neighboring sites.

To do this, we first assign $N+1$ independent random numbers, $X_{j}^{A}(0 \leq j \leq N)$, to every molecule (site) A. These random numbers are Gaussian-distributed with mean 0 and variance 1 , $X_{j}^{A} \sim \mathscr{N}(0,1)$. A nonzero mean value of $m$ can be achieved by replacing $E^{A}$ by $E^{A}+m$. 
We now express the site energy of a molecule $A$ as

$$
E^{A}=\sqrt{a} X_{0}^{A}+\sum_{j=1}^{N} \sqrt{\frac{b_{j}}{\ell_{j}^{A}}} \sum_{B \in \mathscr{S}\left(r_{j}, A\right)} X_{j}^{B}
$$

Here $\mathscr{S}\left(r_{j}, A\right)$ is a sphere of radius $r_{j}$ centered at a molecule $A$, the sum runs over the sites $B \neq A$ located within this sphere, $\ell_{j}^{A}=\frac{4}{3} \pi r_{j}^{3} \rho$ is the number of sites (molecules) in this sphere, and $\rho$ is the site number density (molecules per volume), which we assume to be constant. This is shown schematically in Figure Figure 4. The first term in Equation (Eq. (4)) is used to adjust the width of the final distribution $(\sigma)$, while the second term, which mixes in the energies of the neighbors, is used to adjust the decay of the correlation function.

The choice of energies in the form provided by Equation (Eq. (4)) allows to obtain a simple analytic expression for the correlation function and the variance of the resulting Gaussian distribution. Following the definition of site energies, Equation (Eq. (4)), we can rewrite the expectation value as

$$
\begin{aligned}
\mathbb{E}\left[E^{A} E^{B}\right] & =\sum_{i=1}^{N} \sum_{j=1}^{N} \sqrt{\frac{b_{i} b_{j}}{\ell_{i}^{A} \ell_{i}^{B}}} \sum_{C \in \mathscr{S}\left(r_{i}, A\right)} \sum_{D \in \mathscr{S}\left(r_{j}, B\right)} \delta_{C D} \delta_{i j} \\
& =\sum_{i=1}^{N} \frac{b_{i}}{\sqrt{\ell_{i}^{A} \ell_{i}^{B}}} \sum_{C \in \mathscr{S}\left(r_{i}, A\right) \cap \mathscr{S}\left(r_{i}, B\right)} 1 \\
& =\sum_{i=1}^{N} b_{i} \frac{\ell_{i}^{A B}}{\sqrt{\ell_{i}^{A} \ell_{i}^{B}}}=\sum_{i=1}^{N} \xi\left(\frac{d}{2 r_{i}}\right) b_{i} .
\end{aligned}
$$

Here we used the fact that the random numbers $X_{i}^{A}$ are independent, hence $\mathbb{E}\left[X_{i}^{A} X_{j}^{B}\right]=\delta_{i j} \delta_{A B}$. $\ell_{i}^{A B}=\rho V^{A B}$ is the number of molecules in the intersection of two spheres at a separation $d$, $\mathscr{S}\left(r_{i}, A\right) \cap \mathscr{S}\left(r_{i}, B\right), V^{A B}$ is the volume of this intersection, $V^{A B}=\frac{1}{12} \pi\left(4 r_{i}+d\right)\left(2 r_{i}-d\right)^{2}$ for $d \leq 2 r_{i}$ and zero otherwise (see Figure Figure 4). 
The standard deviation of $X^{A}$ can be evaluated in a similar fashion, yielding

$$
\sigma^{2}=\mathbb{E}\left[\left(E^{A}\right)^{2}\right]-\mathbb{E}\left[E^{A}\right]^{2}=a+\sum_{i=1}^{N} b_{i}
$$

Inserting these expressions into the definition of the correlation function, Equation (Eq. (1)), we obtain the spatial correlation function for molecules $A$ and $B$ at separation $d$

$$
c(d)=\frac{1}{\sigma^{2}} \sum_{j=1}^{N} \xi\left(\frac{d}{2 r_{j}}\right) b_{j}
$$

where $\xi(x)$ is a finite-support function,

$$
\xi(x)= \begin{cases}1-\frac{3}{2} x+\frac{1}{2} x^{3}, & x \leq 1 \\ 0, & x>1\end{cases}
$$

and $\sigma^{2}$ is the variance of site energies,

$$
\sigma^{2}=a+\sum_{j=1}^{N} b_{j}
$$

An intuitive geometric interpretation of Equation (Eq. (7)) is that $\xi(d / 2 r)$ provides a (normalized) overlap of two spheres of radii $r$, with their centers separated by a distance $d$. This overlap determines the spatial correlation function, since sites belonging to the overlap contribute equally to site energies of two molecules located in the centers of the spheres (see Figure Figure 4).

By providing the reference correlation function in $N$ points, $c_{i}^{\mathrm{ref}}=c^{\mathrm{ref}}\left(d_{i}\right), i=1,2, \ldots, N$, we obtain $N$ linear equations

$$
c_{i}^{\mathrm{ref}}=\frac{1}{\sigma^{2}} \sum_{j=1}^{N} \xi_{i j} b_{j}
$$

where $\xi_{i j}=\xi\left(d_{i} / 2 r_{j}\right)$. The solution of Equation (Eq. (10)) provides the weighting coefficients $b_{j}$ and thus a unique interpolation of the reference correlation function in terms of finite support 
functions $\xi$, i.e, piecewise-defined cubic polynomials. Once the coefficients $b_{i}$ are known, $a$ can be determined from Equation (Eq. (9)). In practice, however, some coefficients $b_{i}$ can be negative, leading to imaginary values of $\sqrt{b_{i}}$ in Equation (Eq. (4)) and thus unphysical energies. In this case a recursive scheme, which provides an approximate solution to Equation (Eq. (10)), becomes more practical.

To devise the recursive scheme, we note that there is still a certain degree of flexibility in choosing the grid points $d_{i}$ and $r_{j}$. We now choose the second grid such that $r_{i}=d_{i+1} / 2$, where $d_{i}$ are the points in which the reference function is evaluated, $i=1 \ldots N-1$, and $r_{N}=\left(d_{N}+\Delta\right) / 2$, with $\Delta=d_{N}-d_{N-1}$. Note that the generated correlation function will be zero in the last point, $c\left(2 r_{N}\right)=0$. Given this choice of grid points, $\xi_{i j}=0$ for $i<j$, i.e., $\xi_{i j}$ becomes a triangular matrix. Equation (Eq. (10)) then simplifies to

$$
\sigma^{2} c_{i}^{\mathrm{ref}}=b_{i} \xi_{i i}+\sum_{j=i+1}^{N} \xi_{i j} b_{j}
$$

and can now be solved for $b_{i}$ recursively, starting from $b_{N}$. The final recursive algorithm reads

1. Evaluate $b_{N}=\frac{\sigma^{2}}{\xi_{N N}} c_{N}^{\text {ref }}$.

2. Starting from $N-1$ evaluate recursively for $N-1 \geq i \geq 1$

$$
b_{i}=\frac{1}{\xi_{i i}}\left(\sigma^{2} c_{i}^{\mathrm{ref}}-\sum_{j=i+1}^{N} \xi_{i j} b_{j}\right)
$$

If $b_{i}$ becomes negative, rescale $b_{i+1}$ by a factor $0<\eta<1$ and recalculate $b_{i}$. If $b_{i+1}<\delta$, set it to zero.

3. Evaluate $a$ according to Equation (Eq. (9)).

4. For $M$ sites and $N$ values of the reference function $c_{i}^{\text {ref }}$ generate $M \times(N+1)$ random variables $X_{i}^{A} \sim \mathscr{N}(0,1), 1 \leq A \leq M, 0 \leq i \leq N$.

5. Evaluate site energies of all molecules according to Equation (Eq. (4)). 
Note that the second step includes an $a d$ hoc way of enforcing all $b_{i}$ coefficients to be positive.

\section{Validation}

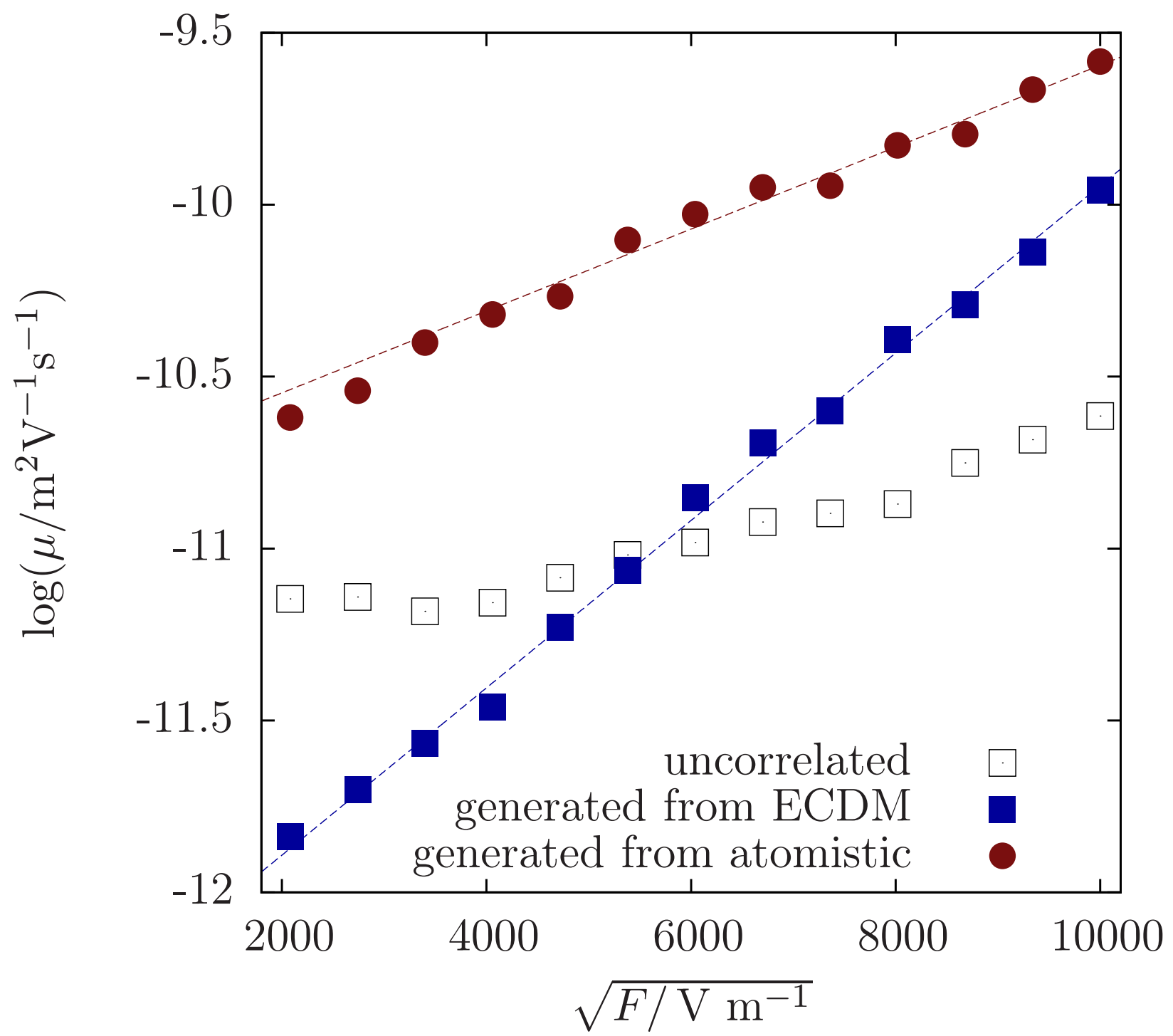

Figure 5: Poole-Frenkel plot for charge transport simulated in a box with identical positions and distributions of site energies but different spatial correlations. A lattice constant of $1.06 \mathrm{~nm}$ has been used in the ECDM.

We now apply the developed scheme and study hole transport in an amorphous morphology of DPBIC. The reference spatial correlation function has been evaluated in the atomistic system of 4000 molecules as described in sec. Section 2. To improve the numerical stability of the algorithm, 
we first smoothen the reference data which is evaluated in $N=24$ points and fit a stretched exponential, $\alpha \exp \left(-\beta d^{\gamma}\right)$, to the atomistic correlation $(\alpha=2.83, \beta=-1.73, \gamma=0.56)$. The fitted function is then used as an input for the algorithm.

Figure Figure 3 shows an excellent agreement between correlations functions evaluated in the atomistic reference and in the generated stochastic system of 40000 sites. We have used the value of $\delta=10^{-5}$ and the scaling factor $\eta$ in the range between 0.8 and 0.99 , which yields sufficient accuracy and fast convergence of the recursive algorithm. For comparison we also show the correlation function of the $\mathrm{ECDM}, c^{\mathrm{ECDM}}(d)=0.74 a / d$, for two different values of the lattice constant $a$. The lattice constant of $1.06 \mathrm{~nm}$ corresponds to the average intermolecular distance of the nearest neighbors and leads to a significant overestimation of the correlation. $a=0.44 \mathrm{~nm}$ is obtained by fitting the charge mobility of an atomistic reference to the analytical expression provided by the ECDM. ${ }^{17}$ The rationale behind a much smaller fitted lattice constant is now apparent: the only adjustable parameter of the ECDM which enters the correlation function is the lattice spacing $a$. Since the field dependence of the charge carrier mobility is very sensitive to spatial correlations of site energies, the ECDM tries to provide the best approximation to the atomistic correlation function - by reducing the lattice spacing to an unphysical value. equation Finally, we shall ask ourselves whether an accurate reproduction of the spatial correlations is important. To answer this question we have performed charge transport simulations in systems of 40000 sites, with the positions of molecules obtained using the iterative Boltzmann inversion method, which reproduces the radial distribution function of the atomistic reference. ${ }^{17,39}$ To this end, we have simulated charge transport in three systems, all with Gaussian-distributed $(\sigma=0.176 \mathrm{eV})$ site energies. The site energies were (i) uncorrelated (ii) correlated with the correlation function of the atomistic reference (iii) correlated with the ECDM correlation function and lattice constant $a=1.06 \mathrm{~nm}$. Since the EGDM site energy correlation is very long-ranged, the (non-uniform) grid in the algorithm was extended to $15 \mathrm{~nm}$. Charge transport simulations were performed at a concentration of $10^{-4}$ carriers per site (four charges), which leads to finite size effects smaller than $0.5 \%{ }^{40}$ The Poole-Frenkel plots, i.e., the logarithm of the mobility versus the square root of the external field, are shown in 
Figure Figure 5 for all three cases. It can be seen that wrong spatial correlations result in ca. two orders of magnitude difference in the mobility values. Furthermore, the slopes also differ, which can be rationalized in terms of the "effective" energetic disorder, i.e., the energy variation of those sites which are most frequently occupied by charge carriers. ${ }^{41}$ We conclude that the spatial correlation of site energies should be reproduced as accurate as possible in order to achieve quantitative modeling of transport in amorphous organic materials.

\section{Discussion and conclusions}

To summarize, the proposed algorithm allows to generate charge transport networks with millions of molecules, which helps to reduce finite-size effects and to study systems of small (but physically relevant) charge densities. One can, therefore, simulate current-voltage characteristics of realistic devices by first tabulating mobility values as a function of temperature, charge density and external electric field and then solving continuous drift-diffusion equations. This approach yields results which are in an excellent agreement with experimental measurements. ${ }^{42}$

It is also possible to extend the scheme to two or more molecule types. In this case one has to take into account not only the autocorrelation function but also the correlation between two different types of molecules and add an additional index for the molecule type to the $b$ coefficients and random numbers.

The described algorithm also improves the moving-average scheme proposed earlier. ${ }^{16}$ It can reproduce arbitrary correlation functions, is easy to automatize, and does not need iterative generation of site energies for trial correlation functions.

To conclude, we have developed a method which can generate Gaussian-distributed energies with a predefined spatial correlation function. We have illustrated that the correlated and extended correlated Gaussian disorder models are unable to reproduce spatial correlations of atomistic systems, or use effective (and unphysical) values of the lattice constant to do this. The developed method can be used to either refine the family of Gaussian disorder models or to construct accurate 
stochastic off-lattice models.

\section{Acknowledgement}

This work was partially supported by Deutsche Forschungsgemeinschaft (DFG) under the Priority Program "Elementary Processes of Organic Photovoltaics" (SPP 1355), BMBF grant MESOMERIE (FKZ 13N10723) and MEDOS (FKZ 03EK3503B), and DFG program IRTG 1404. The project has received funding from the NMP-20-2014 - "Widening materials models" program under grant agreement No. 646259 (MOSTOPHOS). We are grateful to Jens Wehner, Anton Melnyk, Carl Poelking, Christoph Scherer, and Tristan Bereau for a critical reading of the manuscript.

\section{References}

(1) Miller, A.; Abrahams, E. Phys. Rev. 1960, 120, 745-755.

(2) Bässler, H. Phys. Status Solidi B 1993, 175, 15-56.

(3) Bouhassoune, M.; Mensfoort, S. v.; Bobbert, P.; Coehoorn, R. Organic Electronics 2009, 10, $437-445$.

(4) Baranovski, S.; Rubel, O. Description of Charge Transport in Amorphous Semiconductors. In Charge Transport in Disordered Solids with Applications in Electronics; Baranovski, S., Ed.; John Wiley \& Sons, Ltd, 2006; pp 49-96.

(5) Baranovskii, S. D. Phys. Status Solidi B 2014, 251, 487-525.

(6) Novikov, S. V.; Dunlap, D. H.; Kenkre, V. M.; Parris, P. E.; Vannikov, A. V. Phys. Rev. Lett. 1998, $81,4472-4475$.

(7) Young, R. H. Philosophical Magazine Part B 1995, 72, 435-457.

(8) Gemünden, P.; Poelking, C.; Kremer, K.; Andrienko, D.; Daoulas, K. C. Macromolecules 2013, 46, 5762-5774. 
(9) Gemünden, P.; Poelking, C.; Kremer, K.; Daoulas, K.; Andrienko, D. Macromol. Rapid Commun. 2015, 36, 1047-1053.

(10) Dunlap, D. H.; Parris, P. E.; Kenkre, V. M. Phys. Rev. Lett. 1996, 77, 542-545.

(11) Kirkpatrick, J.; Nelson, J. J. Chem. Phys. 2005, 123, 084703.

(12) Rühle, V.; Lukyanov, A.; May, F.; Schrader, M.; Vehoff, T.; Kirkpatrick, J.; Baumeier, B.; Andrienko, D. J. Chem. Theory. Comput. 2011, 7, 3335-3345.

(13) Nelson, J.; Kwiatkowski, J. J.; Kirkpatrick, J.; Frost, J. M. Accounts Chem. Res. 2009, 42, $1768-1778$.

(14) Brédas, J.-L.; Beljonne, D.; Coropceanu, V.; Cornil, J. Chem. Rev. 2004, 104, 4971-5004.

(15) Brédas, J. L.; Calbert, J. P.; Filho, D. A. d. S.; Cornil, J. Proc. Natl. Acad. Sci. USA 2002, 99, 5804-5809.

(16) Baumeier, B.; Stenzel, O.; Poelking, C.; Andrienko, D.; Schmidt, V. Phys. Rev. B 2012, 86, 184202.

(17) Kordt, P.; van der Holst, J. J. M.; Al Helwi, M.; Kowalsky, W.; May, F.; Badinski, A.; Lennartz, C.; Andrienko, D. Adv. Funct. Mater. 2015, 25, 1955-1971.

(18) Novikov, S. V.; Vannikov, A. V. J. Phys. Chem. 1995, 99, 14573-14576.

(19) Kordt, P.; Stenzel, O.; Baumeier, B.; Schmidt, V.; Andrienko, D. J. Chem. Theory. Comput. 2014, 10, 2508-2513.

(20) Strohriegl, P.; Wagner, D.; Schrögel, P.; Hoffmann, S. T.; Köhler, A.; Heinemeyer, U.; Münster, I. Novel host materials for blue phosphorescent OLEDs. SPIE Proceedings, 2013; p 882906.

(21) May, F.; Baumeier, B.; Lennartz, C.; Andrienko, D. Phys. Rev. Lett. 2012, 109, 136401. 
(22) Jorgensen, W. L.; Tirado-Rives, J. J. Am. Chem. Soc. 1988, 110, 1657-1666.

(23) Jorgensen, W. L.; Maxwell, D. S.; Tirado-Rives, J. J. Am. Chem. Soc. 1996, 118, 1122511236.

(24) Besler, B. H.; Merz, K. M.; Kollman, P. A. J. Comput. Chem. 1990, 11, 431-439.

(25) Singh, U. C.; Kollman, P. A. J. Comput. Chem. 1984, 5, 129-145.

(26) Berendsen, H. J. C.; Postma, J. P. M.; Gunsteren, W. F. v.; DiNola, A.; Haak, J. R. J. Chem. Phys. 1984, 81, 3684-3690.

(27) Hutchison, G. R.; Ratner, M. A.; Marks, T. J. J. Am. Chem. Soc. 2005, 127, 2339-2350.

(28) Marcus, R. A. Rev. Mod. Phys. 1993, 65, 599-610.

(29) Ewald, P. P. Ann. Phys. 1921, 369, 253-287.

(30) Smith, E. R. Proc. R. Soc. A 1981, 375, 475-505.

(31) Thole, B. Chem. Phys. 1981, 59, 341-350.

(32) van Duijnen, P. T.; Swart, M. J. Phys. Chem. A 1998, 102, 2399-2407.

(33) Poelking, C.; Andrienko, D. J. Chem. Theory. Comput. 2015, submitted.

(34) Baumeier, B.; Kirkpatrick, J.; Andrienko, D. Phys. Chem. Chem. Phys. 2010, 12, 11103.

(35) Huang, J.; Kertesz, M. J. Chem. Phys. 2005, 122, 234707.

(36) Valeev, E. F.; Coropceanu, V.; da Silva Filho, D. A.; Salman, S.; Brédas, J.-L. J. Am. Chem. Soc. 2006, 128, 9882-9886.

(37) Gillespie, D. T. J. Comput. Phys. 1976, 22, 403-434.

(38) Gillespie, D. T. J. Phys. Chem. 1977, 81, 2340-2361. 
(39) Rühle, V.; Junghans, C.; Lukyanov, A.; Kremer, K.; Andrienko, D. J. Chem. Theory. Comput. 2009, 5, 3211-3223.

(40) Kordt, P.; Speck, T.; Andrienko, D. Phys. Rev. B 2015, submitted.

(41) Schrader, M.; Körner, C.; Elschner, C.; Andrienko, D. J. Mater. Chem. 2012, 22, 2225822264.

(42) Kordt, P.; Stodtmann, S.; Badinski, A.; Helwi, M. A.; Lennartz, C.; Andrienko, D. Phys. Chem. Chem. Phys. 2015, 17, 22778-22783. 


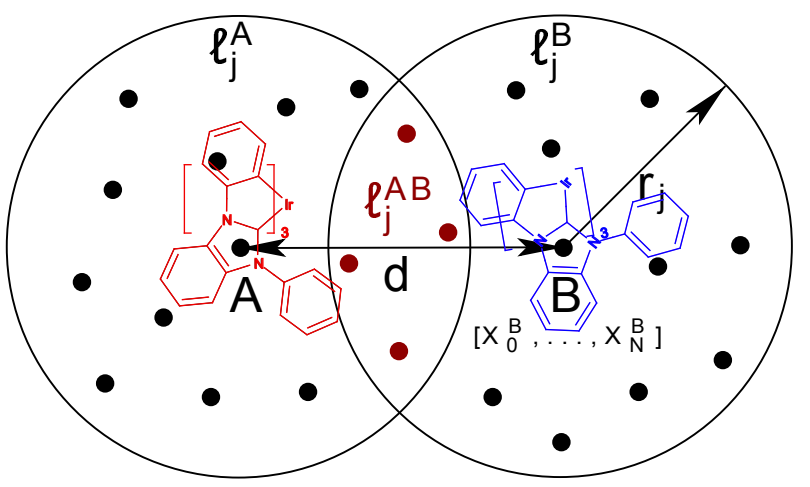

Figure 6: Table of Content figure 